\title{
Squared-field amplitude modulus and radiation intensity nonequivalence within nonlinear slabs
}

\author{
Alberto Lencina ${ }^{1}$ and Pablo Vaveliuk ${ }^{2}$ \\ ${ }^{1}$ Departamento de Física, Centro de Ciências Exatas e da Natureza, \\ Universidade Federal da Paraíba, Caixa Postal 5008 CEP 58051-970, João Pessoa, PB Brazi \\ ${ }^{2}$ Departamento de Física, Universidade Estadual de Feira de Santana, CEP 44031-460, Feira de Santana, BA Brazil
}

(Dated: November 13, 2018)

\begin{abstract}
This paper presents a novel approach to wave propagation inside the Fabry-Perot framework. It states that the time-averaged Poynting vector modulus could be nonequivalent with the squaredfield amplitude modulus. This fact permits the introduction of a new kind of nonlinear medium whose nonlinearity is proportional to the time-averaged Poynting vector modulus. Its transmittance is calculated and found to differ with that obtained for the Kerr medium, whose nonlinearity is proportional to the squared-field amplitude modulus. The latter emphasizes the nonequivalence of these magnitudes. A space-time symmetry analysis shows that the Poynting nonlinearity should be only possible in noncentrosymmetric materials.
\end{abstract}

PACS numbers: 42.25.Bs, 42.65.Pc, 42.65.Hw

\section{INTRODUCTION}

A classical topic in electromagnetism is the study of wave transmission in a finite parallel-plane faces medium, know as Fabry-Perot resonator. When the medium presents nonlinear behavior, bistability appears [1]. To explain this phenomenon, the nonlinear Fabry-Perot resonator (NLFP) was modelled by a third order susceptibility or Kerr-type nonlinearity [2]. At monochromatic plane wave excitation, the NLFP stationary regime is summarized in a non-time-dependent nonlinear wave equation for the complex field amplitudes, the nonlinear Helmholtz equation (NLHE). Hence, the reflectance and transmittance problem reduces to finding the NLHE solution with appropriate boundary conditions for the field amplitude modulus and phase.

The NLHE complexity led to approximate methods of resolution. Many approaches consider two counterpropagating waves in the medium and the analysis is done by separately considering the effects on each wave $[2,3,4,5]$. Unfortunately, the linear superposition principle is no longer valid in nonlinear media and the separation of the electromagnetic field in these back and forth waves is meaningless. As a result, the NLHE separation into two equations, one for each wave, is only possible by neglecting various coupling nonlinear terms that would give an important contribution to the accuracy of the transmittance results. Moreover the Slowly Varying Envelope Approximation (SVEA) is often applied to these waves $[2,3,4]$ when, yet within the counter-propagating wave approach, its validity was questioned [5]. Also, the boundary conditions were simplified rather than rigorously treating them [2, 6]. The above facts suggest that all these approximated approaches could not physically

\footnotetext{
*Corresponding author A. L.: agl@fisica.ufpb.br
}

be equivalents to the exact problem.

The work done by Chen and Mills exactly solve the NLFP for an absorptionless Kerr-type medium [7]. The proper of its resolution method was to assume a general complex field within the medium, disregarding the concept of counter-propagating waves. Chen and Mills derive a two coupled equation system for the field amplitude modulus and phase together with general boundary conditions, thus obtaining an analytic-transcendental solution for the transmittance of the NLFP.

On the other hand, their work permitted us to note an implicit difference between the time-averaged Poynting vector modulus, i.e. the electromagnetic radiation intensity $I$, and the squared-field amplitude modulus $\left(|E|^{2}\right)$ inside the nonlinear medium. If the nonequivalence of these magnitudes were true, it could change certain wellestablished fundamental concepts in classical electrodynamics. This fact motivated us to develop a novel approach to wave propagation in nonlinear media inside the Fabry-Perot framework called S-Formalism. It introduces a new variable related to the time-averaged Poynting vector which states that its magnitude could be nonequivalent with the squared-field amplitude modulus contrary to commonly accepted. Furthermore, the S-Formalism presents two important advantages: it permits to directly monitor the radiation intensity within the medium, and it avoids approximations, such as the SVEA, simplification of boundary conditions, and so on.

The fact that the time-averaged Poynting vector modulus be nonequivalent with the squared-field amplitude modulus, as the S-Formalism will show, implies that the nonlinearity of Kerr-type media is not proportional to the intensity which is contrary to what has been established to-date. This assertion leads to the following question regarding the modelling of the NLFP: is it a Kerr-type nonlinearity, or does it vary proportionately to the intensity? As this question does not have a definitive answer, the existence of the latter cannot be denied. Then, we define the Poynting medium as the medium where nonlinear- 


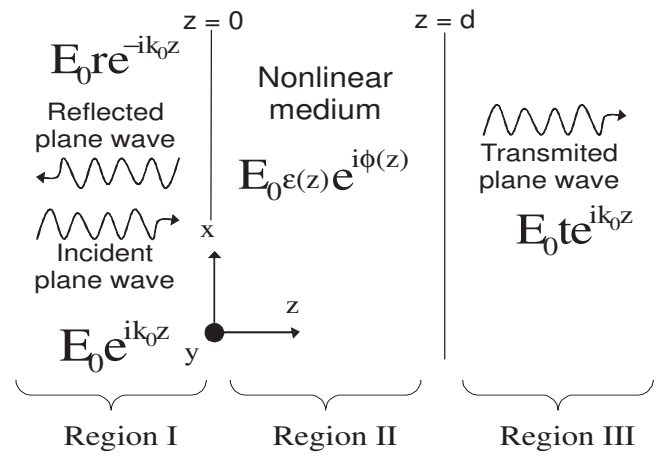

FIG. 1: An harmonic plane wave strikes a nonlinear FabryPerot resonator, to be reflected and transmitted. The Regions I and III constitute, for simplicity, the same linear dielectric medium (e.g., air).

ity is proportional to intensity. Thus, our objective is to solve the Poynting-NLFP through the S-Formalism comparing the resultant transmittance with that obtained for a $K e r r-N L F P$ to remark the nonequivalence between squared-field amplitude modulus and radiation intensity.

In section II, we derive the S-Formalism in the following form: first the time-averaged Poynting vector assuming harmonic fields is calculated. Then, a dimensionless variable which is proportional to intensity is introduced. Thus, a set of field evolution differential equations and the general boundary condition equations on the new field variable are obtained, which constitutes the S-Formalism. In section III, the new approach is applied to derive the transmittance for the Poynting-NLFP and the results compared with that obtained for a KerrNLFP. A brief discussion about the possibily of existence and observation of Poynting media is given. Finally, in section IV, we conclude.

\section{THE S-FORMALISM APPROACH}

Refering to Figure 1 we start writing the linearly polarized transversal harmonic electromagnetic fields of frequency $\omega$ as

$$
\begin{aligned}
& \mathbf{E}_{\ell}(z, t)=\frac{1}{2}\left(E_{\ell}^{\omega}(z) e^{-i \omega t}+\text { c.c. }\right) \hat{\mathbf{i}}, \\
& \mathbf{H}_{\ell}(z, t)=\frac{1}{2}\left(H_{\ell}^{\omega}(z) e^{-i \omega t}+\text { c.c. }\right) \hat{\mathbf{j}},
\end{aligned}
$$

where $E_{\ell}^{\omega}(z)$ and $H_{\ell}^{\omega}(z)$ are the non-time-dependent complex amplitudes for $\ell=$ I, II, III. From region I, a plane wave of amplitude $E_{0}$ and wave vector $k_{0}$ impinges perpendicularly on a nonmagnetic, isotropic and spatially nondispersive medium of thickness $d$ (region II). The optical field is assumed to maintain its polarization along this region so that a scalar approach is valid. The reflected and transmitted plane waves have amplitudes $r E_{0}$ and $t E_{0}$ with $r$ and $t$ the complex reflection and transmission coefficients, respectively. Then, at region I and
III the spatial-dependent complex amplitudes are given by

$$
\begin{aligned}
E_{I}^{\omega}(z) & =E_{0}\left(e^{i k_{0} z}+r e^{-i k_{0} z}\right), \\
E_{I I I}^{\omega}(z) & =E_{0} t e^{i k_{0} z} .
\end{aligned}
$$

Similarly to Ref. 7], at region II, we write down the following ansatz for the spatial-dependent complex amplitude of the electric field:

$$
E_{I I}^{\omega}(z)=E_{0} \mathcal{E}(z) e^{i \phi(z)}
$$

where the dimensionless amplitude $\mathcal{E}(z)$, and phase $\phi(z)$, are both real functions of $z$.

The time-averaged Poynting vector $\left\langle\mathbf{E}_{\ell}(z, t) \times \mathbf{H}_{\ell}(z, t)\right\rangle$ can be easily calculated with the aid of Faraday's law, giving

$$
\langle\mathbf{S}\rangle_{\ell}=\frac{1}{2 \mu_{0} \omega} \operatorname{Im}\left\{\left[E_{\ell}^{\omega}(z)\right]^{*} \frac{\partial E_{\ell}^{\omega}(z)}{\partial z}\right\} \hat{\mathbf{k}},
$$

where $\mu_{0}$ is the vacuum permeability. From this expression we calculate the intensity for the three regions:

$$
\begin{aligned}
\langle S\rangle_{I} & =I_{0}\left(1-|r|^{2}\right) \\
\langle S\rangle_{I I} & =I_{0} k_{0}^{-1} \mathcal{E}^{2}(z) \frac{\partial \phi(z)}{\partial z} \equiv I_{0} S(z), \\
\langle S\rangle_{I I I} & =I_{0}|t|^{2}
\end{aligned}
$$

where $I_{0}=k_{0} E_{0}^{2} /\left(2 \mu_{0} \omega\right)$ is the incident intensity. In region II, Eq. (6b) defines the dimensionless field variable

$$
S \equiv k_{0}^{-1} \mathcal{E}^{2} \frac{\partial \phi}{\partial z}
$$

directly related with the intensity inside the medium and which will characterize the S-Formalism. From Eq. (7) it is clear that if $\phi$ is not a linear function on $z$, as is often happens in nonlinear media, then $S$ and $\mathcal{E}^{2}$ are nonequivalents.

The next step is to derive the NLHE in terms of the classical field variables $(\mathcal{E}, \phi)$, and transform it into a set of equivalent equations in terms of $(\mathcal{E}, S)$. The NLHE is derived from the macroscopic Maxwell equations complemented by appropriate constitutive relations. We assume that the polarization $\mathbf{P}$, and current density $\mathbf{J}$, vary only in the electric field direction with frequency $\omega$, neglecting higher harmonics, and their spatial-dependent complex amplitudes satisfy the following constitutive relations:

$$
\begin{aligned}
& P_{I I}^{\omega}(z)=\epsilon_{0} \chi_{g e n}\left[z, E_{I I}^{\omega}, H_{I I}^{\omega}\right] E_{I I}^{\omega}(z), \\
& J_{I I}^{\omega}(z)=\sigma_{g e n}\left[z, E_{I I}^{\omega}, H_{I I}^{\omega}\right] E_{I I}^{\omega}(z),
\end{aligned}
$$

where $\epsilon_{0}$ is the vacuum permittivity and, $\chi_{g e n}$ and $\sigma_{g e n}$ are the generalized susceptibility and conductivity respectively, that are real and contain the linear as well as a possible nonlinear medium response. Note that the constitutive relations are not explicitly written since cases 
could exist where it is not possible to describe the nonlinear polarization and current density by the classical electric field power expansion. Thereby, the scalar NLHE is

$$
\left[\frac{d^{2}}{d z^{2}}+k_{0}^{2}\left(1+\chi_{g e n}\right)+i \omega \mu_{0} \sigma_{g e n}\right] E_{I I}^{\omega}(z)=0 .
$$

This equation constitutes the starting point to study several linear and nonlinear monochromatic wave propagation phenomena within the Fabry-Perot framework. Substituting Eq. (4) into (10) and by using Eq. (7), we derive the following set of spatial evolution equations for the field variables $\mathcal{E}(z)$ and $S(z)$ :

$$
\begin{aligned}
& \frac{d^{2} \mathcal{E}}{d z^{2}}+k_{0}^{2}\left(\left(1+\chi_{g e n}[z, \mathcal{E}, S]\right) \mathcal{E}-\frac{S^{2}}{\mathcal{E}^{3}}\right)=0,(11 \\
& \frac{d S}{d z}+\frac{\omega}{k_{0}} \mu_{0} \sigma_{g e n}[z, \mathcal{E}, S] \mathcal{E}^{2}=0
\end{aligned}
$$

To guarantee the physical content of the solution, these equations must be necessarily complemented with the following boundary conditions: the continuity of the tangential components of the electric and magnetic field at the interfaces. The general boundary conditions were rigourously derived in Ref. [7]: four equations as functions of $(\mathcal{E}, \phi)$ at $z=0$ and $z=d$ which, by using Eq. (77), are transformed into three equations in terms of the variables $(\mathcal{E}, S)$ to give

$$
\begin{aligned}
& \left(\mathcal{E}(0)+\frac{S(0)}{\mathcal{E}(0)}\right)^{2}+\left(\left.\frac{1}{k_{0}} \frac{d \mathcal{E}}{d z}\right|_{z=0}\right)^{2}=4, \\
& S(d)-\mathcal{E}^{2}(d)=0 \\
& \left.\frac{d \mathcal{E}}{d z}\right|_{z=d}=0
\end{aligned}
$$

From Eqs. (6) and (12), the transmittance is obtained as

$$
T=|t|^{2}=S(d),
$$

and the energy conservation is guaranteed thorough the expression:

$$
|r|^{2}+|t|^{2}=1-(S(0)-S(d)) .
$$

This equation establishes that the reflectance and transmittance are limited by the boundary values of the timeaveraged Poynting vector. For a nonabsorbent medium $S(d)=S(0)$, then $|r|^{2}+|t|^{2}=1$.

Equations (11) and (12) represent the "S-Formalism" which were derived without assuming approximations such as the counter-propagating waves, SVEA, simplifications on the boundary conditions, and so on. Also, note that Eq. 11b represents the time-averaged Poynting Theorem applied to the problem of harmonic fields simplifying the interpretation of $\sigma_{g e n}$ as the dissipation properties of the medium. In particular, when $\sigma_{\text {gen }}=0$, the dimensionless intensity $S$ is a constant fixed by the boundary conditions. Furthermore, through $S(z)$ it is possible to monitor directly the intensity along the medium as a function of the spatial coordinate, as opposed to using the conventional formalism.

The S-Formalism is useful to analyze the linear case as well as the nonlinear one. Before to study the latter, i.e. the comparison between the Poynting and Kerr media in an effort to show the explicit difference between $S$ (or $I$ ) and $\mathcal{E}^{2}$ (or $\left|E^{2}\right|$ ) in nonlinear media, we refer to the linear case. According to our analysis [8], there are only two situations where the relationship $I=c t e\left|E^{2}\right|$ holds true: firstly, a single plane wave propagates in a infinite or semi-infinite linear dielectric characterized by $\sigma_{g e n}=0$ and $\chi_{g e n}=\chi^{(1)}$ where $\chi^{(1)}$ is the linear susceptibility. Under these conditions, Eqs. (11) relate the constants $S$ and $\mathcal{E}$ by $S=\left(1+\chi^{(1)}\right)^{1 / 2} \mathcal{E}^{2}$; secondly, a single plane wave propagates in a semi-infinite linear absorber characterized by $\chi_{g e n}=\chi^{(1)}$ and $\sigma_{g e n}=\sigma$ where $\sigma$ is the ohmic conductivity and such that $S(z) \propto \mathcal{E}^{2}(z)$, being both proportional to a decreasing exponential function of $z$. On the contrary, when the medium is finite, e.g. a Fabry-Perot with boundary conditions at interfaces, $S$ is no longer equivalent to $\mathcal{E}^{2}$, not even for the linear dielectric case because $S$ is a constant and $\mathcal{E}^{2}$ is an oscillating function of $z[8]$.

\section{THE POYNTING MEDIUM}

\section{A. Constitutive relations and transmittance results}

At this point, we introduce the Poynting medium by the following constitutive relations:

$$
\begin{aligned}
& \chi_{\text {gen }}=\chi^{(1)}+\gamma I_{0} S(z), \\
& \sigma_{\text {gen }}=0,
\end{aligned}
$$

where $\gamma$ is the nonlinear coefficient. Eqs. (11) have a simple analytical solution given by

$S(z)=S_{0}$,

$\mathcal{E}(z)=\sqrt{\frac{S_{0}}{2}\left[\left(1-\frac{k_{0}^{2}}{k_{1}^{2}}\right) \cos \left[2 k_{1}(z-d)\right]+1+\frac{k_{0}^{2}}{k_{1}^{2}}\right]}$

where $\gamma>0$ and $k_{1}^{2}=k_{0}^{2}\left(1+\chi^{(1)}+\gamma I_{0} S_{0}\right)$. The constant $S_{0}$ is fixed by

$$
\left(1-\frac{k_{1}^{2}}{k_{0}^{2}}\right) \mathcal{E}^{2}(0)+\left(3+\frac{k_{1}^{2}}{k_{0}^{2}}\right) S_{0}-4=0 .
$$

Combining Eqs. (18) and (19), the transmitance can be expressed in a similar fashion as the linear Fabry-Perot resonator as

$$
T=\frac{1}{1+F \sin ^{2}\left(k_{1} d\right)},
$$




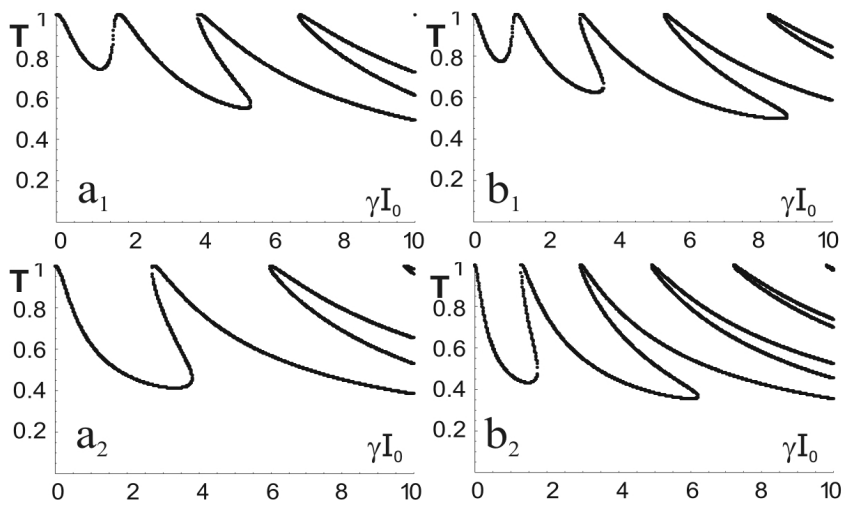

FIG. 2: Transmittance against nonlinear parameter for $\left(a_{i}\right)$ Poynting medium, $\left(b_{i}\right)$ Kerr medium with $k_{0} d=2 \pi$. For $i=1, \chi^{(1)}=1.25$; and $i=2, \chi^{(1)}=5.25$.

where $F=k_{0}\left(1-k_{1} / k_{0}\right)^{2} /\left(4 k_{1}\right)$. Carefully noting that Eq. (20) is a transcendental expression since $k_{1}$ depends on $S_{0}$.

Now, we compare the transmittance results of the Poynting and Kerr media. The latter defined by

$$
\begin{aligned}
\chi_{\text {gen }} & =\chi^{(1)}+\gamma I_{0} \mathcal{E}^{2}(z), \\
\sigma_{\text {gen }} & =0 .
\end{aligned}
$$

The Kerr-NLFP transmittance results were taken from Ref. 7]. Figure 2 shows $T$ against the nonlinear parameter $\gamma I_{0}$ for two different values of $\chi^{(1)}$. Figures $2\left(a_{i}\right)$ and $2\left(b_{i}\right)$ correspond to the Poynting and Kerr medium, respectively. From these figures, it is apparent that the transmittance of the Poynting-NLFP as well as the KerrNLFP are multistable. However, for increasing values of $\chi^{(1)}$, the peak transmittance separation diminishes for the Kerr medium while it increases for the Poynting medium. Also, the Kerr multistability appears for smallest values of the nonlinear parameter $\gamma I_{0}$. The transmittance difference of both media emphasizes the $I$ and $|E|^{2}$ nonequivalence. Figure 3 depicts $T$ on the dimensionless thickness $k_{0} d\left(1+\chi^{(1)}\right)^{1 / 2} /(2 \pi)$ enhancing the nonlinearity difference of Poynting and Kerr media. Note that the departure from Airy-type function for the Kerr medium is stronger than for the Poynting medium.

On the other hand, Figure 4 shows that the Poynting nonlinear susceptibility, $\chi_{g e n}-\chi^{(1)}$, has a constant value along the medium ( $z$ coordinate). In return, the Kerr

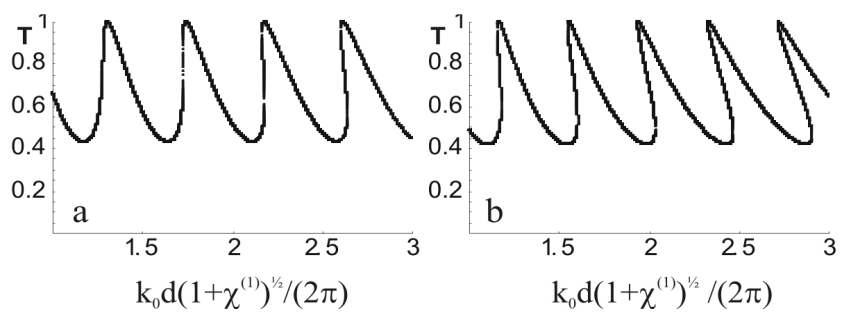

FIG. 3: Transmittance against dimensionless thickness for (a) Poynting medium, (b) Kerr medium with $\chi^{(1)}=5.25$ and $\gamma I_{0}=2$.

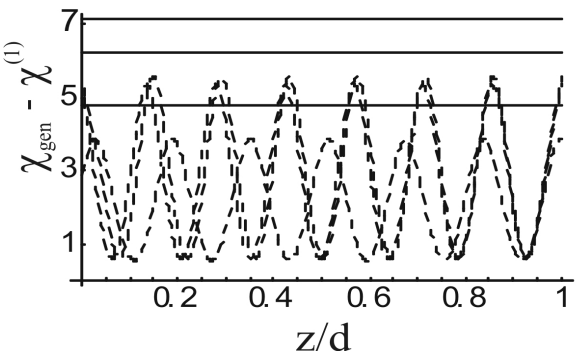

FIG. 4: Nonlinear susceptibility against dimensionless spatial coordinate for each of the three solutions compatible with the boundary conditions. Continuous line: Poynting medium. Broken line: Kerr medium. The parameter values are $\gamma I_{0}=9, \chi^{(1)}=1.25$, and $k_{0} d$ as defined in Fig. 2 .

nonlinear susceptibility varies periodically. This fact implies the formation of a phase grating in the Kerr medium on the contrary to the Poynting medium. Perhaps, this substantial difference can be measured, and this could be the starting point to experimentally identify a Poynting medium.

\section{B. Transformation properties under spatial inversion and time reversal}

It is a fact that several unusual types of nonlinearities were predicted before its experimental observation, as it was remarked, for example, in the pioneer works of Baranova et al. 9]. With the aim to elucidate the isotropic medium requirements to observe the new phenomena, those authors pointed out the necessity of a analysis about transformation properties of electromagnetic quantities under rotations, spatial inversion and time reversal. Therefore, this symmetry analysis is also necessary to delimit the Poynting medium requirements.

The magnitude that characterizes the electromagnetic response of a Poynting medium is their nonlinear susceptibility $\chi^{(P)}$ which is linear on the time-averaged Poynting vector $\langle\mathbf{S}\rangle$, as follows from the constitutive relations [Eq. [15]]. In a general form, it can be written as

$$
\chi_{i j}^{(P)}=\gamma_{i j k}\langle\mathbf{S}(\mathbf{r}, t)\rangle_{k},
$$

with $i, j, k=x, y, z$ and

$$
\langle\mathbf{S}(\mathbf{r}, t)\rangle_{k}=\frac{1}{T} \int_{t}^{t+T}\left[\mathbf{E}\left(\mathbf{r}, t^{\prime}\right) \times \mathbf{H}\left(\mathbf{r}, t^{\prime}\right)\right]_{k} d t^{\prime},
$$

where $\mathbf{E}$ and $\mathbf{H}$ are harmonics of period $\tau=2 \pi / \omega$ and time interval $T \gg \tau$. The susceptibility tensor transforms as even under spacial inversion $(r \rightarrow-r)$ and time reversal $(t \rightarrow-t)$, contrary to the Poynting vector and its time-averaged which transforms odd under spatial inversion and time reversal, i.e. $\langle\mathbf{S}(\mathbf{r}, t)\rangle \rightarrow-\langle\mathbf{S}(-\mathbf{r}, t)\rangle$ and $\langle\mathbf{S}(\mathbf{r}, t)\rangle \rightarrow-\langle\mathbf{S}(\mathbf{r},-t)\rangle$, respectively [10]. Then, a medium possessing a linear connection between $\chi_{i j}$ and 
$\langle S\rangle_{z}$ should be noninvariant with respect to spatial inversion and time reversal. Otherwise, the space-time symmetry will be violated in the constitutive relation [Eq. (23)].

The lack of parity symmetry under inversion of coordinates is proper of materials without inversion center, i. e. Poynting nonlinearity should be only possible in noncentrosymmetric materials. There are several materials candidates to possess a Poynting nonlinearity, as for example the cubic crystals with zincblended structure such as GaAs, InSb and others. In these materials intensity-dependent transmission and bistability was experimentally observed [1]]. Also, isotropic homogeneous liquids formed by nonracemic mixtures or solutions of mirror-asymmetric (chiral) molecules with strong nonlinear optical susceptibility, as product of several nonlinear processes [12], are also feasible of posses a Poynting nonlinearity. In addition, parity under time reversal should be violated in Poynting media. This means that any weak dissipative process, that converts field energy into heat, is necessary to remove the rule relating to the $t \rightarrow-t$ transformation. For example, either very weak absorbtion or current flow by external quasi-static field, that basically do not affect the wave propagation at light frequency $\omega$, would ensure the medium non-invariance under time reversal.

We believe that, in spite of experimental works are required, the above preliminary analysis could stimulate further discussions regarding the existence of Poynting media.

\section{CONCLUSIONS}

In summary, we derive a new formalism in terms of dimensionless variables related with the time-averaged Poynting vector and field amplitude modulus within the Fabry-Perot framework. The S-Formalism shows explicitly that the energy intensity and squared-field amplitude modulus are only equivalents for a single plane wave propagating in a linear infinite or semi-infinite medium. Otherwise, they are nonequivalents. Besides, the SFormalism presents two important advantages: it permits to directly monitor the time-averaged Poynting vector in the medium and it avoids approximations, such as SVEA, simplification of the boundary conditions, and so on. To emphasize this nonequivalence we introduce the Poynting medium, whose nonlinearity is proportional to the intensity instead of the electric squared-field amplitude modulus such as in the Kerr medium. We find marked disagreement in the transmittance of both media, which support the differences between I and $|E|^{2}$. Also, a space-time symmetry analysis shows that the Poynting nonlinearity should be only possible in noncentrosymmetric materials.

The statements and analysis pointed out here constitute an advance on theoretical views of basic concepts in electrodynamics. The S-Formalism could be important in problems where the time-averaged Poynting vector must be rigourously monitored like in photoconductor or photorefractive materials. Further to this particular case studied here, this new approach leaves open the possibility of new physical results in actual topics on nonlinear wave propagation such as spatial solitons, wave mixing and others. Finally, we leave open the possibility that experimental techniques, based on intensity dependent phase changes of a Gaussian beam such as Z-Scan Technique [13], could not truly measure Kerr-type nonlinearity. On the contrary, they could be measuring a Poynting-type instead.

\section{Acknowledgments}

The authors thank Prof. Boris Ya. Zel'dovich and anonymous Referee for the suggestions about symmetry properties of Poynting media. The authors also thank Victor Waveluk for valuable advices. A. L. thanks to CLAF-CNPq fellowship.
[1] H. M. Gibbs, S. L. McCall and T. N. C. Venkatesan, Phys. Rev. Lett 36, 1135 (1976).

[2] J. H. Marburger and F. S. Felber, Phys. Rev. A 17, 335 (1978).

[3] D. A. B. Miller, IEEE J. Quantum Electron.QE-17, 306 (1981).

[4] J. Danckaert et al., Opt. Commun. 71, 317 (1989).

[5] B. Biran, Opt. Commun. 78, 183 (1990).

[6] K. Fobelets and K. Thielemans, Phys. Rev. A 53, 4400 (1996).

[7] W. Chen and D.L. Mills, Phys. Rev. B 35, 524 (1987); 38, 12814 (1988).

[8] A. Lencina, B. Ruiz and P. Vaveliuk (unpublished).

[9] N. B. Baranova, Yu. V. Bogdanov and B. Ya. Zel'dovich, Sov. Phys. Usp. 20, 870 (1977); Opt. Commun. 22, 243 (1977).
[10] J. D. Jackson, Classical Electrodynamics, (Wiley, New York 1999), 3rd. ed., p. 271.

[11] H. M. Gibbs et al., Phylos. Trans. R. Soc. London A 313 , 245 (1984); D. A. B. Miller, S. D. Smith and A. Johnston, Appl. Phys. Lett. 35, 658 (1979).

[12] N. I. Koroteev, Kvantovaya Elektron. 21, 1063 (1994).

[13] M. Sheik-Bahae et al., IEEE J. Quantum Electron. QE26, 760 (1990). 